\title{
DISCOVERY OF THE NEW SLOWLY PULSATING B STAR HD 163830 (B5 II/III) FROM MOST SPACE-BASED PHOTOMETRY
}

\author{
C. Aerts,${ }^{2,3}$ P. De Cat,${ }^{4}$ R. Kuschnig, ${ }^{5}$ J. M. Matthews, ${ }^{5}$ D. B. Guenther,${ }^{6}$ A. F. J. Moffat,${ }^{7}$ \\ S. M. Rucinski, ${ }^{8}$ D. Sasselov, ${ }^{9}$ G. A. H. Walker, ${ }^{10}$ and W. W. Weiss ${ }^{11}$ \\ Received 2006 February 2; accepted 2006 March 28; published 2006 April 20
}

\begin{abstract}
We report the discovery of a new slowly pulsating B star, with the largest number of detected frequencies to date by more than a factor of 3, based on 37 days of MOST (Microvariability and Oscillations of STars) satellite guide star photometry. The star HD $163830(V=9.3, \mathrm{~B} 5 \mathrm{II} / \mathrm{III})$ varies in 20 detected frequencies in the range 0.035-1.06 day $^{-1}(0.4-12.3 \mu \mathrm{Hz})$ with amplitudes from 0.7 to $7.6 \mathrm{mmag}$ (with a signal-to-noise ratio from 4 to 41). Eighteen of these frequencies are consistent with low-degree, high-order nonradial $g$-modes of seismic models of an evolved $4.5 M_{\odot}$ star. We are unable to identify one unique model due to lack of mode identifications. The lowest two frequencies may be associated with the rotation of HD 163830, but firm proof of this must await future spectroscopic data.
\end{abstract}

Subject headings: stars: early-type — stars: fundamental parameters — stars: individual (HD 163830) — stars: oscillations - stars: rotation

Online material: color figure

\section{INTRODUCTION}

Slowly pulsating B (SPB) stars (Waelkens 1991) show great promise for asteroseismology. Their $g$-mode pulsations should be sensitive probes of their deep stellar interiors, since $g$-mode amplitudes remain large right into the core (unlike $p$-modes whose amplitudes are significant only in the outer layers). But these stars also present serious observational and theoretical challenges. (1) Their pulsation periods are of order a few days, making them difficult to disentangle in ground-based data due to cycle/day aliasing. (2) Their theoretical eigenspectra are so rich (Dziembowski et al. 1993; Gautschy \& Saio 1993) that one cannot simply find a unique match to a model by fitting the observed frequencies unless there is prior information about the degrees and orders of the modes. (3) Rotation is a serious complication for mode identification in these stars because rotational splitting is large enough to cause multiplets of adjacent radial orders to overlap. The same great promise and obstacles exist for other $g$-mode pulsators on the lower main sequence, like the $\gamma$ Doradus variables.

\footnotetext{
${ }^{1}$ Based on data from the MOST satellite, a Canadian Space Agency mission, jointly operated by Dynacon Inc., the University of Toronto Institute for Aerospace Studies, and the University of British Columbia, with the assistance of the University of Vienna.

${ }^{2}$ Institute of Astronomy, University of Leuven, Celestijnenlaan 200 B, B3001 Leuven, Belgium; conny@ster.kuleuven.be.

${ }^{3}$ Department of Astrophysics, Radboud University Nijmegen, P.O. Box 9010, 6500 GL Nijmegen, Netherlands.

${ }^{4}$ Royal Observatory of Belgium, Ringlaan 3, B-1180 Brussels, Belgium.

${ }^{5}$ Department of Physics and Astronomy, University of British Columbia, 6224 Agricultural Road, Vancouver, BC V6T 1Z1, Canada.

${ }^{6}$ Department of Astronomy and Physics, St. Mary's University, Halifax, NS B3H 3C3, Canada.

${ }^{7}$ Département de Physique, Université de Montréal, CP 6128, Succursale Centre-Ville, Montréal, QC H3C 3J7, Canada.

${ }^{8}$ David Dunlap Observatory, University of Toronto, P.O. Box 360, Richmond Hill, ON L4C 4Y6, Canada.

${ }^{9}$ Harvard-Smithsonian Center for Astrophysics, 60 Garden Street, Cambridge, MA 02138.

${ }^{10} 1234$ Hewlett Place, Victoria, BC V8S 4P7, Canada.

${ }^{11}$ Institut für Astronomie, Universität Wien, Türkenschanzstrasse 17, A1180 Wien, Austria.
}

Huge long-term ground-based observing campaigns (see, e.g., De Cat \& Aerts 2002 and references therein for the SPBs, and Henry et al. 2005 and references therein for the $\gamma$ Dor stars) had been successful in reliably discovering multiperiodic variables. But the Hipparcos mission (Perryman et al. 1997) demonstrated the power of space-based observations to expand our understanding of $g$-mode pulsators on the upper main sequence. By mining the Hipparcos photometric archive, Waelkens et al. (1998) and Handler (1999) discovered many previously unknown SPB and $\gamma$ Dor stars, respectively.

However, despite these efforts, the maximum number of well-established independent frequencies to be found in ground-based data of single SPB or $\gamma$ Dor stars is five (see De Cat \& Aerts 2002 and Poretti et al. 2002). This paucity of observed frequencies compared to the densely populated theoretical eigenspectra has made it difficult to even address issues like rotational splitting. Here we report the detection of 20 frequencies in a new SPB star discovered by the MOST space mission. The WIRE satellite has meanwhile also revealed numerous new SPB candidates with about five to 15 frequencies (H. Brüntt et al. 2006, in preparation).

\section{OBSERVATIONS AND DATA REDUCTION}

HD 163830 was one of 20 guide stars in the field for the MOST primary science target WR 103, observed in 2005 June. This field also contains the guide star HD 163868 (B5 Ve), in which the discovery by MOST of a rich $g$-mode eigenspectrum made it the first in a new class of SPBe stars (Walker et al. 2005). The basic aspects of the guide star photometry in this field and the data reduction procedure are already described by Walker et al. (2005). A constant star (at the level of precision of the measurements), HD 164388 (A2 IV, $V=7.9$ ), was adopted as a comparison for the HD 163868 photometry, and it is also used as a stable reference for HD 163830 in this Letter.

HD 163830 was monitored for 36.6 days from 2005 June 14 to July 21 , with about 137,000 signal values in the original time series. Data were collected for only about 50 minutes of each 101 minute orbit, since the target field is outside the MOST Continuous Viewing Zone and was eclipsed by the Earth for half of each orbit. The final duty cycle of the time series is 
about $38 \%$, after filtering of outliers and discarding data contaminated by excess cosmic-ray hits during satellite passages through the South Atlantic Anomaly in the Earth's magnetosphere. For the eventual frequency analysis, the data were binned into 2 minute samples, giving a total of 9890 points in the final light curve. The MOST light curve of HD 163830 is presented as dots in Figure 1. This plot illustrates that the MOST data set far exceeds any previous photometric data of known SPBs both in duty cycle and quality.

The exposure time of the star tracker was set to $1.5 \mathrm{~s}$. As in the Direct Imaging section of the MOST Science CCD (see Rowe et al. 2006), the point-spread function (PSF) of the images has an FWHM of about 2 pixels, where the focal plane scale is about $3^{\prime \prime}$ pixel $^{-1}$. Only preselected rasters of the CCD are read out, each $40 \times 40$ pixels in size, with a guide star at the center. The CCD parameters are bias 550 ADU, gain $\sim 20$ e $\mathrm{ADU}^{-1}$, and read noise $2.5 \mathrm{ADU}_{\text {pixel }}^{-1} \mathrm{rms}$. A maximum of 20 such rasters can be chosen for a single guide field, due to downlink and onboard software limitations. The brightness limits for guide stars range from 6.5 to $10.5 \mathrm{mag}$.

Each raster/image is preprocessed automatically onboard. First, an instantaneous background reading is determined by averaging the signals of the first and last rows of the raster. This subtracts much of the stray light present in the image effectively, even if the scattered earthshine changes dramatically from exposure to exposure. Then a fixed threshold value of 20 ADU is added to the background; this number is subtracted from the signal from each individual pixel. The threshold is applied to avoid introducing spurious signals into the attitude control data processing. As a consequence, the contributions of the tails of the stellar PSF are not included in the photometry. The fact that no images are downloaded from the guide star data and the fact that only the highest signal pixels are included in the measurements combine to limit the photometric precision to 2-2.5 times the photon noise limit. However, the long uninterrupted time series still provides low noise level results for relatively faint guide stars such as HD 163830.

Finally, all pixels with a positive residual signal value (essentially the core pixels of the PSF) are summed, representing a single intensity measurement per exposure. Residual background due to scattered earthshine not subtracted by the onboard procedure is filtered on the ground as described by Walker et al. (2005).

The MOST star tracker CCD must generally be operated at a sampling rate higher than the science $\mathrm{CCD}$, in order to update the Attitude Control System. In the case of the primary science target WR 103, science measurements were obtained at $10 \mathrm{~s}$ intervals, during which five guide star exposures were accumulated. These five values were added, and the cumulative signal was stored in the MOST Science Data Stream, so the effective guide star sampling interval is also $10 \mathrm{~s}$.

\section{FREQUENCY ANALYSIS}

We performed a Fourier time series analysis using Scargle's (1982) algorithm, successively identifying the strongest peaks and removing them from the light curve to search for additional frequencies in the residuals. The spectral window of these data is so clean due to the lack of long or periodic gaps in the time series (see Fig. 1) that aliasing is not a concern. The prewhitening was done by performing a least-squares fit to find the most likely value of the amplitude, phase, and frequency, using the height and position of the peak from the Scargle periodogram as a starting value. The errors derived in this way are formal errors that do not take into account the correlation of the noise or the

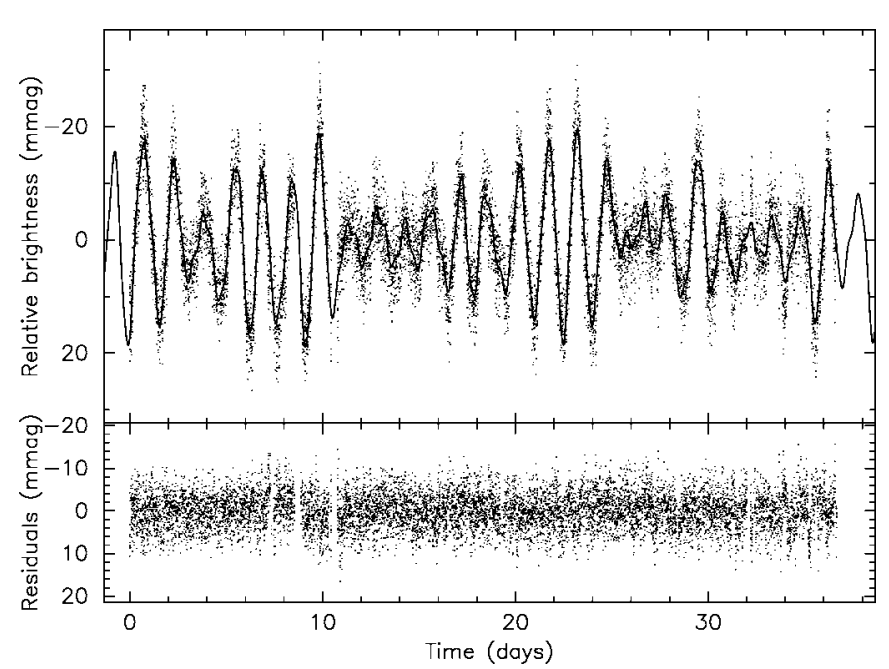

FIG. 1.-Top: Observed light curve of HD 163830 (dots) compared with the final fit given in eq. (1) (solid line). Bottom: Residuals after subtraction of the final fit.

dependence among the parameters. Hence, they are an underestimation of the true errors (Schwarzenberg-Czerny 1991).

From this process, we identified 21 frequencies with amplitudes greater than a 3.9 signal-to-noise ratio $(\mathrm{S} / \mathrm{N})$, where the $\mathrm{S} / \mathrm{N}$ was estimated as the average peak amplitude in a Scargle periodogram obtained after final prewhitening, in the range $0-2$ day $^{-1}$ where power was detected (see Breger et al. 1993). This is considered to be a rather strict significance criterion for the known sample of SPBs (De Cat \& Cuypers 2003). One of the identified frequencies, 2.0018 day $^{-1}$, is a known artifact in MOST data arising due to modulation of stray earthshine during the satellite's Sun-synchronous dusk-dawn orbit (see Reegen et al. 2006). The detection of 20 independent wellseparated frequencies within $[0,1.1]$ day $^{-1}$ was possible thanks to the high $\mathrm{S} / \mathrm{N}$ level of the peaks and the sinusoidal waveform of the modes. The satellite orbital frequency peak occurs at 14.2 day $^{-1}$, i.e., well outside the frequency range seen in the target.

Scargle periodograms at a few different stages of prewhitening (omitting the 2.0018 day $^{-1}$ artifact) are shown in Figure 2. The final fit to the MOST light curve of HD 163830 (including the artifact) is defined by

$$
y_{i}=a+\sum_{j=1}^{21} b_{j} \sin \left[2 \pi\left(f_{j} t_{i}+\phi_{j}\right)\right]
$$

where the values for the free parameters $a, b_{j}, f_{j}$, and $\phi_{j}$ are given in Table 1. The solid curve in Figure 1 corresponds to this solution. The residuals after subtraction of this fit have a standard deviation of $4.20 \mathrm{mmag}$ and are also provided in Figure 1. Note that most of the peaks listed in Table 1 have an amplitude well below this value. Indeed, the $\mathrm{S} / \mathrm{N}$ in the Scargle periodogram of the residuals amounts to only 0.18 mmag mainly thanks to the reduction factor $\sqrt{9890}$.

There is a hint of additional low-amplitude quasi-periodic variability in the residuals. All the candidate frequencies found in them have an amplitude below $3.6 \mathrm{~S} / \mathrm{N}$, however, so it is dangerous to make further identifications or to assign formal errors from continued prewhitening. We ended the frequency identification conservatively when the formal $\mathrm{S} / \mathrm{N}$ dropped below 3.9, having high confidence in the frequencies and amplitudes in Table 1. 


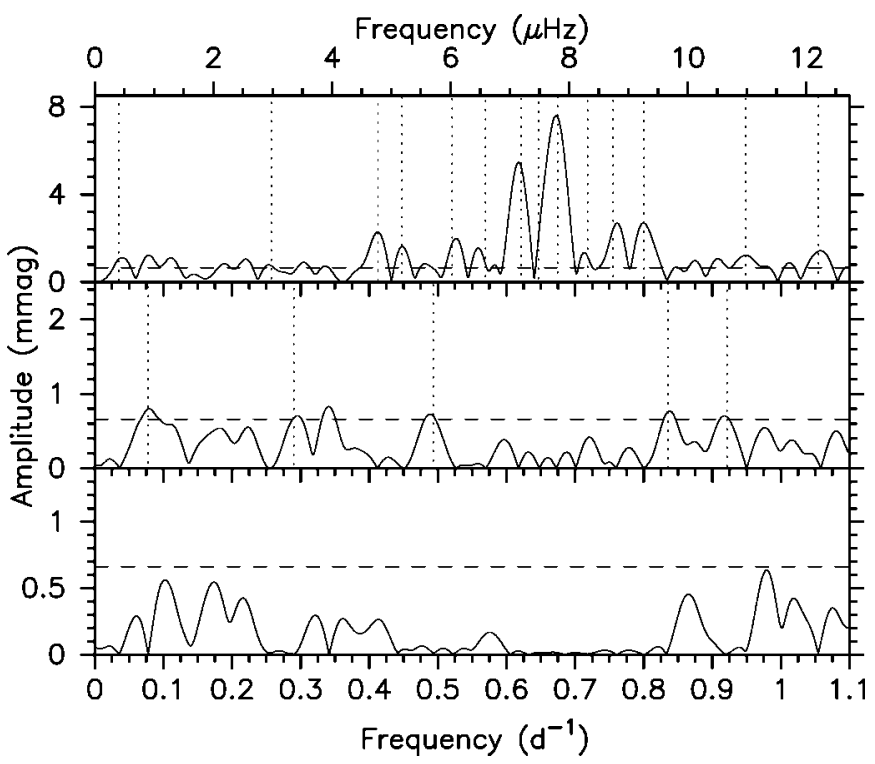

FIG. 2.-Scargle periodogram of the MOST data of HD 163830 (top panel), after prewhitening with the 15 dominant frequencies (middle panel) and after prewhitening with the 21 frequencies listed in Table 1 (bottom panel). The dotted vertical lines indicate the frequency values listed in Table 1 from leastsquares fitting. The dashed horizontal lines indicate the $3.6 \mathrm{~S} / \mathrm{N}$ level. Note the different scale of the $y$-axes. The stray light frequency at 2.0039 day $^{-1}$ is omitted for clarity.

\section{SEISMIC INTERPRETATION}

One high-quality archival seven-color Geneva measurement is available for HD 163830. We used it to derive the effective temperature and gravity of the star following the method by Künzli et al. (1997). This resulted in $T_{\text {eff }}=13,700 \pm 500 \mathrm{~K}$ and $\log g=3.79 \pm 0.14$ dex, which are compatible with the spectral type and luminosity class of B5 II/III assigned by Houk (1982). As there is no parallax estimate available for HD 163830, we interpolated in standard models computed with the stellar evolution code CLÉS (Scuflaire 2005) and found a mass of $4.5 \pm 0.4 M_{\odot}$.

To compare the detected oscillation frequencies of HD 163830 with those predicted by theory in the approximation of a nonrotating B-type star, we computed seismic models from the zero-age main sequence (ZAMS) until core exhaustion using CLÉS for different stellar masses and for $X=0.71$, $Z=0.015$, a mixing-length parameter of 1.75 times the pressure scale height, and no core overshooting. We plot these models in a $\left(\log T_{\text {eff }}, \log g\right)$-diagram in Figure 3 . The observational error box of HD 163830 is also shown, as well as the SPB instability strip for modes of $l=1,2,3$. Subsequently, we computed the frequencies of the zonal modes of all the $M=4.5 M_{\odot}$ models on the main sequence for $l=1,2,3$ and tested the excitation of these modes with the nonadiabatic code MAD (Dupret 2001). The outcome of these instability computations is presented in Figure 4 for the $l=1,2$ modes. The spectrum for the $l=3$ modes is even denser and is omitted here for brevity. We also show the observed frequencies (except the stray light frequency and the lowest ones of 0.035 and 0.079 day $^{-1}$ ) and the upper limit of the effective temperature of HD 163830. It can be seen that there are numerous $l=$ 1, 2 modes excited in these models and that they can explain well the observed frequencies of HD 163830, particularly since we only show the zonal modes in Figure 4 . We thus interpret the variability of our target in terms of high-order low-degree nonradial $g$-modes as expected in SPBs. Given the expected
TABLE 1

Light-Curve Solution of HD 163830, ACCORDing to the 21 TERMS GIVEN IN EQ. (1) BESIDES $a=921.56(5) \mathrm{mmag}$

\begin{tabular}{llcllr}
\hline \hline Frequency & \multicolumn{1}{c}{$\begin{array}{c}f_{j} \\
\left.\text { day }^{-1}\right)\end{array}$} & $\begin{array}{c}f_{j} \\
(\mu \mathrm{Hz})\end{array}$ & $\begin{array}{c}b_{j} \\
(\mathrm{mmag})\end{array}$ & \multicolumn{1}{c}{$\phi_{j}$} & $\mathrm{~S} / \mathrm{N}$ \\
\hline$f_{1} \ldots \ldots \ldots$ & $0.6744(5)$ & $7.806(5)$ & $7.6(2)$ & $0.125(8)$ & 41.4 \\
$f_{2} \ldots \ldots \ldots$ & $0.621(2)$ & $7.19(2)$ & $5.5(5)$ & $0.34(3)$ & 30.0 \\
$f_{3} \ldots \ldots \ldots$ & $0.647(4)$ & $7.48(4)$ & $2.8(4)$ & $0.46(5)$ & 15.3 \\
$f_{4} \ldots \ldots \ldots$ & $0.756(1)$ & $8.75(1)$ & $2.38(9)$ & $0.37(3)$ & 13.0 \\
$f_{5} \ldots \ldots \ldots$ & $0.8007(9)$ & $9.27(1)$ & $2.2(1)$ & $0.15(2)$ & 12.0 \\
$f_{6} \ldots \ldots \ldots$ & $0.412(1)$ & $4.77(2)$ & $1.64(8)$ & $0.50(2)$ & 8.9 \\
$f_{7} \ldots \ldots \ldots$ & $0.949(1)$ & $10.980(7)$ & $1.40(6)$ & $0.45(2)$ & 7.6 \\
$f_{8} \ldots \ldots \ldots$ & $0.576(2)$ & $6.67(3)$ & $1.4(6)$ & $0.17(4)$ & 7.6 \\
$f_{9} \ldots \ldots \ldots$ & $1.0546(7)$ & $12.206(9)$ & $1.37(6)$ & $0.53(2)$ & 7.5 \\
$f_{10} \ldots \ldots \ldots$ & $0.521(2)$ & $6.03(2)$ & $1.31(7)$ & $0.53(3)$ & 7.1 \\
$f_{11} \ldots \ldots \ldots$ & $0.0350(8)$ & $0.405(9)$ & $1.18(7)$ & $0.92(2)$ & 6.4 \\
$f_{12} \ldots \ldots \ldots$ & $0.448(2)$ & $5.18(3)$ & $1.17(7)$ & $0.44(4)$ & 6.4 \\
$f_{13} \ldots \ldots \ldots$ & $2.0018(8)$ & $23.17(1)$ & $1.11(6)$ & $0.21(2)$ & 6.0 \\
$f_{14} \ldots \ldots \ldots$ & $0.718(4)$ & $8.31(5)$ & $0.9(1)$ & $0.61(8)$ & 4.9 \\
$f_{15} \ldots \ldots \ldots$ & $0.257(2)$ & $2.98(3)$ & $0.9(1)$ & $0.25(5)$ & 4.9 \\
$f_{16} \ldots \ldots \ldots$ & $0.832(2)$ & $9.63(3)$ & $0.87(8)$ & $0.49(5)$ & 4.5 \\
$f_{17} \ldots \ldots \ldots$ & $0.285(3)$ & $3.30(3)$ & $0.83(9)$ & $0.20(5)$ & 4.5 \\
$f_{18} \ldots \ldots \ldots$ & $0.494(3)$ & $5.72(3)$ & $0.80(7)$ & $0.25(4)$ & 4.4 \\
$f_{19} \ldots \ldots \ldots$ & $0.343(1)$ & $3.97(2)$ & $0.77(6)$ & $0.49(3)$ & 4.2 \\
$f_{20} \ldots \ldots \ldots$ & $0.079(2)$ & $0.91(2)$ & $0.72(6)$ & $0.71(3)$ & 3.9 \\
$f_{21} \ldots \ldots \ldots$ & $0.921(2)$ & $10.66(2)$ & $0.72(7)$ & $0.66(4)$ & 3.9 \\
\hline
\end{tabular}

Notes. - The reference epoch for the phases $\phi_{j}$ is the time of first observation (HJD 2,453,536.33498108). The quoted formal errors underestimate the true variance because they were derived from a least-squares fit ignoring the correlation of the noise and the dependence among the parameters. Frequency $f_{13}$ is due to the detection of stray light.

large number of excited modes, some of the residual power seen in Figure 1 is probably due to $g$-modes that we have not been able to adequately resolve from one another. We stress that we did not perform a matching between the observed frequencies and the theoretical ones of the models, because we lack information on the $m$-values of the modes and on the stellar rotation frequency. Figure 4 only shows that the observed spectrum is compatible with theoretical models in the appropriate area of the H-R diagram.

The frequencies $f_{11}=0.035 \mathrm{day}^{-1}$ and $f_{20}=0.079 \mathrm{day}^{-1} \approx$ $2 f_{11}$ are clearly of a different character, being an order of magnitude smaller than the other frequencies we have identified. A possible explanation for these frequencies is that they are associated with the star's rotational frequency $\Omega$. Unfortunately, the corresponding period of 28.6 days is only slightly less than the total time coverage of the MOST photometry of HD 163830 , so it is not safe to say whether this variation even repeats periodically. Such low frequencies have also been seen in other

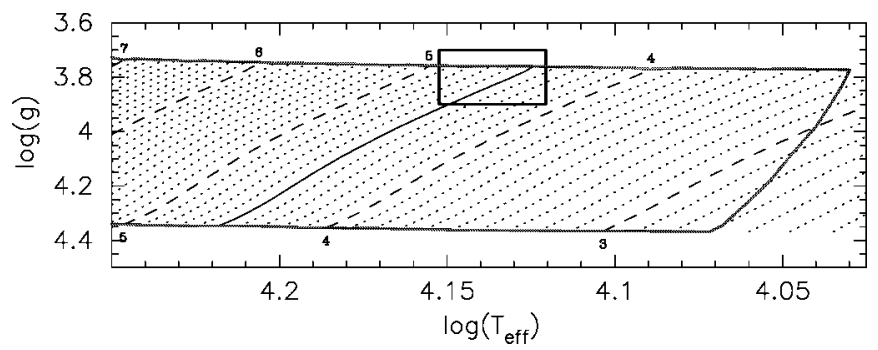

Fig. 3.-Evolutionary models (dashed and dotted lines) in steps of $0.1 M_{\odot}$ computed with CLÉS from the ZAMS until core exhaustion. The masses for the models in dashed lines are given in solar values. The models whose frequency spectrum is given in Fig. 4 are indicated as a solid black line. The observational error box of HD 163830 is represented by the rectangle. The instability strip computed from MAD is indicated by a thick gray line and represents the region in which modes of $l=1,2,3$ are excited. 

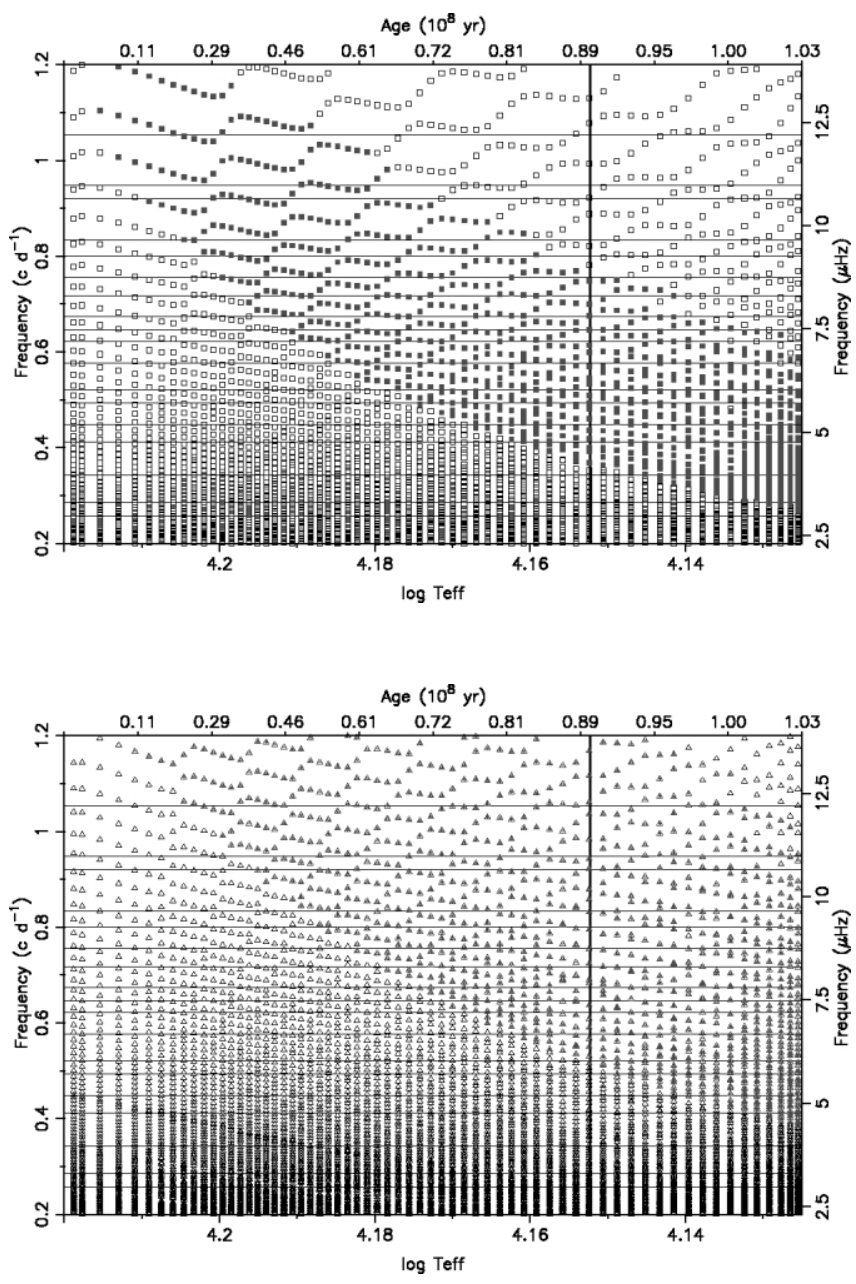

FIG. 4.-Predicted frequencies for $l=1$ (top) and $l=2$ (bottom) modes for main-sequence stellar models with parameters $4.5 M_{\odot}, X=0.71, Z=$ 0.015 , mixing length $\alpha=1.75$ pressure scale heights, and no core overshooting. Excited modes are indicated with filled symbols, and stable modes with open symbols. The observed frequencies of HD 163830 are indicated as full horizontal lines. HD 163830 is situated to the right of the vertical line. [See the electronic edition of the Journal for a color version of this figure.]

SPBs (De Cat \& Aerts 2002) where they are difficult to interpret in terms of pulsation.

Some of the adjacent frequencies of HD 163830 listed in Table 1 show spacings in the range $0.03-0.04 \mathrm{day}^{-1}$, and are compatible with rotational splitting, since high-order $g$-modes produce multiplets with spacings $m[1-1 / l(l+1)] \Omega$. However, without prior mode identifications of $(l, m)$ it is impossible to decide whether these are multiplet components or isolated modes. De Cat et al. (2005) have shown that the oscillation frequencies in the corotating frame of the star can differ substantially from the same frequencies seen in the observer's frame, even in the case of slow rotation and low-degree modes. If $\Omega=0.035 \mathrm{day}^{-1}$, then one would expect adjacent frequencies for $l=1$ to be spaced by only $0.0175 \mathrm{day}^{-1}$; for $l=2$ by $0.029 \mathrm{day}^{-1}$. Yet, as can be seen in Figure 4, even independent eigenfrequencies of adjacent radial order and the same degree in a nonrotating model show separations comparable to this. For these reasons, it is premature to try to find a more specific match to one particular seismic model until there is independent information about the rotation rate of the star or constraints on the mode values, both of which will require spectroscopic follow-up.

\section{SUMMARY}

We discovered 20 intrinsic frequencies in the 37 day MOST light curve of the little-studied B5 II/III star HD 163830. We interpreted 18 of these frequencies in terms of high-order nonradial $g$-modes in an SPB, thus adding HD 163830 to this class of oscillators. It becomes at once the SPB with the largest number of detected oscillation frequencies. We provided a seismic model that is compatible with the observed frequency spectrum. This model is only one of numerous other possibilities. In order to fine-tune our seismic analysis, we need empirical mode identification, either from multicolor photometry or from line profile variations.

Our results illustrate the gain of nearly continuous photometry compared to ground data in disentangling the frequency spectra of the $g$-modes in SPBs. Similar results are to be expected for $\gamma$ Doradus stars. We can therefore anticipate numerous new results from space-based observatories that are capable of gathering long continuous strings of data from space, such as MOST, WIRE, COROT, and Kepler.

C. A. and P. D. C. are indebted to Richard Scuflaire, MarcAntoine Dupret, and Mario Ausseloos for providing their software codes CLÉS, MAD, and SCAN. C. A. is supported by the Research Council of the KU Leuven under grant GOA/2003/04. J. M. M., D. B. G., A. F. J. M., S. M. R., and G. A. H. W. acknowledge funding from the Natural Sciences and Engineering Research Council (NSERC) Canada. R. K.'s work is supported by the Canadian Space Agency. W. W. W. received funding from the Austrian Forschungsförderungsgesellschaft (FFG-ALR) and the Science Fonds (FWF-P17580).

\section{REFERENCES}

Breger, M., et al. 1993, A\&A, 271, 482

De Cat, P., \& Aerts, C. 2002, A\&A, 393, 965

De Cat, P., Briquet, M., Daszyńska-Daszkiewicz, J., Dupret, M. A., de Ridder, J., Scuflaire, R., \& Aerts, C. 2005, A\&A, 432, 1013

De Cat, P., \& Cuypers, J. 2003, in ASP Conf. Ser. 292, Interplay of Periodic, Cyclic and Stochastic Variability in Selected Areas of the H-R Diagram, ed. C. Sterken (San Francisco: ASP), 377

Dupret, M.-A. 2001, A\&A, 366, 166

Dziembowski, W. A., Moskalik, P., \& Pamyatnykh, A. A. 1993, MNRAS, 265,588

Gautschy, A., \& Saio, H. 1993, MNRAS, 262, 213

Handler, G. 1999, MNRAS, 309, L19

Henry, G. W., Fekel, F. C., \& Henry, S. M. 2005, AJ, 129, 2815

Houk, N. 1982, Michigan Spectral Survey (Ann Arbor: Dept. Astron., Univ. Michigan), 3
Künzli, M., North, P., Kurucz, R. L., \& Nicolet, B. 1997, A\&AS, 122, 51 Perryman, M. A. C., et al. 1997, The Hipparcos and Tycho Catalogues (ESA SP-1200) (Noordwijk: ESA)

Poretti, E., et al. 2002, A\&A, 384, 513

Reegen, P., et al. 2006, MNRAS, 367, 1417

Rowe, R., et al. 2006, ApJ, in press

Scargle, J. D. 1982, ApJ, 263, 835

Schwarzenberg-Czerny, A. 1991, MNRAS, 253, 198

Scuflaire, R. 2005, CLÉS User Manual, Ver. 18 (Liège: Univ. Liège)

Waelkens, C. 1991, A\&A, 246, 453

Waelkens, C., Aerts, C., Kestens, E., Grenon, M., \& Eyer, L. 1998, A\&A, 330,215

Walker, G. A. H., et al. 2005, ApJ, 635, L77 